Meta

Journal des traducteurs

Translators' Journal

\title{
Traduire les textes juridiques dans un contexte de plurilinguisme officiel : quelle formation pour quelles compétences spécifiques?
}

\section{Valérie Dullion}

Volume 59, numéro 3, décembre 2014

Traduction et plurilinguisme officiel

Translation and Official Multilingualism

URI : https://id.erudit.org/iderudit/1028661ar

DOI : https://doi.org/10.7202/1028661ar

Aller au sommaire du numéro

\section{Éditeur(s)}

Les Presses de l’Université de Montréal

ISSN

0026-0452 (imprimé)

1492-1421 (numérique)

Découvrir la revue

Citer cet article

Dullion, V. (2014). Traduire les textes juridiques dans un contexte de plurilinguisme officiel : quelle formation pour quelles compétences spécifiques? Meta, 59(3), 636-653. https://doi.org/10.7202/1028661ar

\section{Résumé de l'article}

Question fondamentale pour les systèmes plurilingues, la qualité de la traduction juridique est abordée ici sous l'angle non pas des aménagements institutionnels, mais des acteurs. Les spécificités des contextes de plurilinguisme officiel sont mises en relation avec la problématique de la compétence traductionnelle, de façon à tirer des conclusions applicables à la formation. Dans les systèmes plurilingues, la traduction juridique doit répondre à des exigences particulières liées à l'intertextualité, au statut du texte traduit, à la pluralité des destinataires et à l'institutionnalisation du processus. Ces exigences ont des implications sur plusieurs plans. À cet égard, l'article se réfère aux modèles généraux de compétence et à leur approfondissement interdisciplinaire dans la perspective de la traduction juridique. Il propose d'intégrer la question du plurilinguisme officiel dans un enseignement structuré autour des situations professionnelles, en mettant l'accent sur les aspects suivants : l'utilisation raisonnée des ressources documentaires ; l'acquisition de connaissances relatives au droit linguistique et au fonctionnement institutionnel ; la prévention des interférences et du jargon ; et le développement d'une capacité d'argumenter et d'interagir avec d'autres professionnels. Pour terminer, l'article illustre la singularité des divers contextes plurilingues. Il rejette l'idée selon laquelle ils représenteraient pour la traduction juridique une situation simplifiée, nécessitant des compétences purement techniques.
Ce document est protégé par la loi sur le droit d'auteur. L’utilisation des services d’Érudit (y compris la reproduction) est assujettie à sa politique d'utilisation que vous pouvez consulter en ligne.

https://apropos.erudit.org/fr/usagers/politique-dutilisation/ 


\title{
Traduire les textes juridiques dans un contexte de plurilinguisme officiel : quelle formation pour quelles compétences spécifiques?
}

\author{
VALÉRIE DULLION \\ Université de Genève, Genève, Suisse \\ Valerie.Dullion@unige.ch
}

\section{RÉSUMÉ}

Question fondamentale pour les systèmes plurilingues, la qualité de la traduction juridique est abordée ici sous l'angle non pas des aménagements institutionnels, mais des acteurs. Les spécificités des contextes de plurilinguisme officiel sont mises en relation avec la problématique de la compétence traductionnelle, de façon à tirer des conclusions applicables à la formation. Dans les systèmes plurilingues, la traduction juridique doit répondre à des exigences particulières liées à l'intertextualité, au statut du texte traduit, à la pluralité des destinataires et à l'institutionnalisation du processus. Ces exigences ont des implications sur plusieurs plans. À cet égard, l'article se réfère aux modèles généraux de compétence et à leur approfondissement interdisciplinaire dans la perspective de la traduction juridique. Il propose d'intégrer la question du plurilinguisme officiel dans un enseignement structuré autour des situations professionnelles, en mettant l'accent sur les aspects suivants: I'utilisation raisonnée des ressources documentaires; l'acquisition de connaissances relatives au droit linguistique et au fonctionnement institutionnel; la prévention des interférences et du jargon; et le développement d'une capacité d'argumenter et d'interagir avec d'autres professionnels. Pour terminer, l'article illustre la singularité des divers contextes plurilingues. II rejette l'idée selon laquelle ils représenteraient pour la traduction juridique une situation simplifiée, nécessitant des compétences purement techniques.

\footnotetext{
ABSTRACT

Multilingual systems raise the fundamental question of the quality of legal translation. This article examines the question from the perspective of the actors involved, rather than from that of institutional procedures. Issues specific to contexts of official multilingualism are analysed in relation to problems associated with translation competence, with a view to drawing relevant conclusions for translator training. In multilingual systems, legal translation is subject to particular requirements related to intertextuality, the status of the translated text, the multiplicity of target readers, and the institutionalization of the text production process. These requirements have numerous implications, which are studied here within the framework of general models of competence and their interdisciplinary potential as they pertain to legal translation. It is argued that the question of official multilingualism should be integrated into training based on professional situations by emphasizing the following aspects: the rational use of documentary resources, the acquisition of knowledge relating to language law and the functioning of institutions, the avoidance of linguistic interference and jargon, and the ability to debate and interact with the various stakeholders. In conclusion, the uniqueness of the many different multilingual contexts is highlighted, thereby calling into question the idea that these contexts represent a simplified situation for legal translation construed solely in terms of technical competencies.
} 


\section{MOTS CLÉS/KEYWORDS}

traduction juridique, plurilinguisme officiel, traduction institutionnelle, compétence, formation des traducteurs legal translation, official multilingualism, institutional translation, competence, translator training

\section{Introduction}

Une récente étude sur le plurilinguisme de la législation suisse souligne, dans ses conclusions, le professionnalisme et l'efficacité des traducteurs qui participent au processus d'élaboration des textes. Des éloges qui sont toutefois tempérés par le constat suivant: ces mêmes traducteurs ne parviennent pas toujours à faire entendre leur voix.

Les analyses de [deux lois fédérales] ainsi que de leurs genèses respectives ont révélé le travail précieux et professionnel effectué souvent dans l'urgence par les traductrices et les traducteurs, qui ont notablement contribué à la qualité linguistique globalement élevée des versions linguistiques des lois.

Cette évaluation positive est cependant affectée par le fait que les suggestions et les remarques des traductrices et des traducteurs n'ont malheureusement été prises en compte que de manière insuffisante et peu systématique dans le processus législatif. (Schweizer et Borghi 2011: 415)

$\mathrm{Au}$-delà du contexte spécifique dans lequel elles s'inscrivent, ces observations invitent à s'interroger sur ce qui constitue la compétence d'un traducteur amené à traiter des textes juridiques dans un contexte de plurilinguisme officiel. Question importante, puisque le fonctionnement quotidien des systèmes juridiques plurilingues est tributaire de la qualité des traductions. La qualité est souvent abordée sous le seul angle des aménagements institutionnels (par exemple, corédaction, distinction entre une étape de traduction proprement dite et une étape de révision par des juristes). Le présent article propose de centrer la réflexion sur les acteurs eux-mêmes, à savoir les traducteurs. Pour ce faire, il relie des travaux préexistants portant sur deux objets distincts (section 2): d'une part, les caractéristiques de la traduction juridique dans les contextes de plurilinguisme officiel; d'autre part, les compétences du traducteur et leur développement par une didactique appropriée. Cette démarche vise à mettre en évidence les exigences propres au plurilinguisme officiel (section 3) pour tirer des conclusions applicables à la formation en traduction juridique (section 4). Il s'agira de dégager des principes généraux, mais aussi d'illustrer la nécessité de les relativiser en fonction des réalités locales, chaque système plurilingue ayant ses particularités (section 5). Fondé sur la synthèse de travaux qui reflètent les pratiques de traduction juridique dans divers contextes de plurilinguisme officiel, notre propos a une visée théorique. Il sera toutefois illustré par des exemples concrets empruntés à la Suisse.

Précisons d'emblée que, pour les besoins de cet article, nous définissons le champ de la traduction juridique de façon assez large. Si nous envisageons principalement celle qui est pratiquée en contexte institutionnel (voir Koskinen 2011), le plus souvent par des traducteurs salariés, nous n'excluons pas pour autant d'autres cas de figure. En effet, même pour un indépendant chargé de traduire, par exemple, un contrat pour une entreprise du secteur bancaire, le fait de travailler dans un système juridique 
plurilingue peut avoir certaines implications méthodologiques. C'est vrai en particulier de la recherche documentaire: pour employer une terminologie et une phraséologie appropriées, ce traducteur est amené à se référer à des sources officielles qui préexistent dans la langue cible. Il est aussi amené à évaluer dans quelle mesure telle ou telle formulation a un caractère contraignant et doit impérativement être reprise dans sa propre traduction. En conséquence, les exemples proposés ici relèveront de divers types de discours (législatif, administratif, judiciaire, contractuel) et de situations professionnelles variées.

Rappelons ensuite que, du fait des aménagements institutionnels mentionnés précédemment, il est souvent difficile $\mathrm{d}^{\prime}$ "isoler» la traduction juridique, en la dissociant d'autres modes de transfert linguistique pratiqués dans le domaine du droit. Notre propos ne vise pas à distinguer une traduction juridique «pure» de modalités «hybrides», mises en œuvre surtout dans le domaine législatif (sur cette problématique, voir Šarčević 1997: 105-108). Pour les besoins de cet article, nous estimerons qu'il y a un élément de traduction juridique chaque fois qu'un texte cible est considéré comme étant - au moins de facto - dérivé d'un texte source juridique dont il est censé être l'équivalent. Nous nous plaçons donc sur le plan des modalités concrètes de production des textes juridiques plurilingues, même si, par son statut juridique, le «produit» a parfois valeur de texte original, pleinement authentique, ne portant plus les traces de la traduction. Nous nous plaçons aussi sur le plan des compétences nécessaires à la traduction en tant qu'opération, tout en gardant à l'esprit la complexité sociologique des profils professionnels qui interviennent dans l'élaboration des textes, et l'interaction de ces profils entre eux (voir Borja Albi et Prieto Ramos 2013: 155-202).

\section{Deux thématiques à relier}

\subsection{La traduction juridique dans les contextes de plurilinguisme officiel}

La traduction juridique telle qu'elle se pratique dans les contextes de plurilinguisme officiel a des caractéristiques qui ont déjà été bien étudiées (voir Šarčević 2010; Sandrini 2010). Premièrement, le texte à traduire s'inscrit dans un réseau intertextuel particulièrement dense et cohérent. Cette interdépendance a une conséquence importante pour la terminologie: le traducteur se trouve le plus souvent face à des notions qui ont déjà été uniformisées dans une perspective translinguistique. Son travail consiste non pas à comparer des notions définies différemment à l'intérieur d'ordres juridiques distincts, afin d'établir entre elles une équivalence toujours problématique, mais à formuler un message en faisant appel aux notions d'un seul et même ordre juridique qui s'exprime en plusieurs langues. Deuxièmement, le texte cible lui-même peut avoir une valeur d'instrument juridique. Sa fonction ne se limite donc pas à informer le lecteur sur un acte de communication "étranger». Troisièmement, compte tenu de la diversité des types de textes juridiques qui sont traduits, la traduction ne s'adresse pas toujours à des spécialistes. Elle peut aussi compter parmi ses destinataires le citoyen, le justiciable, l'administré. Le caractère massif de la traduction confère d'ailleurs à cette activité, prise globalement, une influence non négligeable sur la langue et l'évolution de celle-ci. Le risque est d'aboutir, dans le pire des cas, à la généralisation d'un jargon bureaucratique truffé d'interférences. Quatrième- 
ment, la production et la réception des textes sont largement institutionnalisées. La traduction vient ainsi s'insérer dans un processus à caractère collectif, global (comportant un certain nombre d'étapes et d'échelons), interdisciplinaire et plurilingue. Aussi, dans le domaine législatif, le processus aboutissant à des textes qui ne sont pas des «traductions» au sens strict comporte-t-il des phases de traduction et génère la traduction d'autres textes, qu'il s'agisse de versions préliminaires ou partielles, ou encore de documents préparatoires. Quoique peu visibles, les traducteurs sont bien présents, aux côtés d'autres professionnels ${ }^{1}$.

\subsection{Compétence traductionnelle et textes juridiques}

Un certain nombre de modèles généraux ont été proposés pour représenter la compétence du traducteur (voir Kelly 2002: 10-13). Les travaux visant à définir cette compétence emploient en partie des méthodes empiriques ou expérimentales. C'est le cas de ceux menés par le groupe PACTE à partir de 1998 (PACTE 2005). Le groupe a mis en évidence, entre autres, le rôle clé d'une sous-compétence d'ordre stratégique (voir Pacte 2003: 48, 57, 59, 60). Les recherches sur la notion de compétence donnent une assise à la réflexion didactique (Schäffner et Adab 2000), en particulier à la conception d'activités permettant de développer les diverses sous-compétences (voir Kelly 2002: 17-18). Ces recherches trouvent aussi un écho dans la définition de normes professionnelles $^{2}$ ou dans les politiques de formation des traducteurs ${ }^{3}$.

La réflexion sur les compétences nécessaires en traduction spécialisée peut s'appuyer sur ces modèles généraux (voir Scarpa 2001/2008: 249-282). Dans le domaine qui nous occupe, elle les approfondit en y intégrant la relation interdisciplinaire entre droit et traduction (voir Cao 2007: 37-52). Cette démarche prend en considération les fonctions communicatives particulières remplies par les textes juridiques, ainsi que les diverses situations professionnelles dans lesquelles ils sont traduits.

\subsection{Un modèle spécialisé}

Un modèle adapté aux besoins de la traduction juridique a ainsi été proposé par Fernando Prieto Ramos (2011). Précisons ici que ce modèle porte sur la traduction juridique en général, et non spécifiquement dans les contextes de plurilinguisme officiel. Il distingue cinq sous-compétences:

- Stratégique/méthodologique:

Elle se manifeste essentiellement dans la capacité de résoudre des problèmes, par exemple des problèmes terminologiques au terme d'une analyse faisant appel au droit comparé.

- Communicative et textuelle:

Elle consiste notamment à exploiter des connaissances contrastives (dans le couple de langues considéré) sur les différents types de discours juridique.

- Thématique et culturelle:

Elle joue de toute évidence un rôle clé en traduction spécialisée (voir Scarpa 2001/2008: 262-270). S'il est généralement vrai que les traducteurs de textes juridiques ont besoin d'une connaissance du domaine moins poussée que les juristes, il faut surtout relever que l'acquisition de cette connaissance doit être orientée 
autrement, en fonction d'autres priorités (sur ce débat, voir Lavoie 2003). L'objectif est de donner une vue d'ensemble du phénomène juridique assortie de repères suffisants pour une recherche documentaire raisonnée (voir Wiesmann 2009, ainsi que le point suivant). Des composantes telles que le droit comparé et l'argumentation juridique doivent occuper une place proportionnellement plus importante que dans la formation des juristes.

- Documentaire et technologique:

Elle couvre l'utilisation des outils et ressources spécifiques, tels que les bases de données législatives ou terminologiques, ou encore les corpus illustrant des genres textuels. Il ne s'agit pas seulement de connaître ces ressources, mais aussi de savoir les utiliser. Trouver la version actuelle d'une loi est certes devenu très facile. Retrouver le texte exact de tel article en vigueur à telle date peut être nettement plus ardu lorsque des abrogations, modifications et changements de numérotation se sont accumulés au fil du temps. D’autant que les recueils informatisés ne sont pas conçus partout selon les mêmes principes.

- Interpersonnelle et professionnelle:

Elle correspond entre autres à une capacité d'exercer son activité dans un cadre qui est souvent, lui-même, très réglementé. À titre d'exemple, on peut classer sous cette rubrique la connaissance des contraintes propres au travail des traducteurs jurés.

\section{Exigences particulières du plurilinguisme officiel}

Par rapport à ce modèle, quelles sont les exigences particulières du plurilinguisme officiel? Tout d'abord, un renforcement de la sous-compétence documentaire et technologique. Pour assurer la cohérence terminologique et, plus largement, intertextuelle, le traducteur doit rechercher ce qui a déjà été traduit dans la langue cible. Cela ne signifie pas pour autant que la capacité de se documenter remplacerait, dans les contextes de plurilinguisme officiel, les compétences en droit comparé et en résolution de problèmes qui sont habituellement cruciales en traduction juridique. Aussi perfectionnée soit-elle, une banque de terminologie ne fournit pas toujours du «prêtà-l'emploi». Dès que le traducteur se trouve dans des situations d'interaction entre plusieurs ordres de compétence (par exemple, fédéral/provincial, européen/national/ régional) ou de désignation d'une notion nouvelle, il est amené à résoudre des problèmes et donc à mobiliser la sous-compétence stratégique et méthodologique (voir Kjaer 2007: 79; Raus 2010; Tranchant 2011: 201-202, dans le contexte de l'Union européenne).

Ensuite, le statut du texte traduit exige que la portée du message juridique soit restituée avec une grande précision, afin d'assurer une équivalence d'effet (voir Šarčević 1997: 70-72). Un exemple frappant peut être donné ici, qui concerne la formulation des délais. Un texte normatif pérennise le droit de séjour de ressortissants étrangers qui ont été admis dans un pays à titre provisoire, pour des motifs humanitaires. La mesure profite à ces personnes à condition qu'elles soient arrivées au cours d'une période donnée. Or il se trouve qu'en français, "être arrivé avant le 31 décembre [de telle année] » n’a pas la même signification que «être arrivé au plus tard le 31 décembre » : cette dernière formulation équivaut plutôt à «être arrivé avant le $1^{\text {er }}$ janvier [de l'année suivante] ». Dans un contexte de plurilinguisme officiel, une erreur dans le choix entre ces deux formulations peut créer une incertitude bien réelle pour toutes les personnes qui auraient eu le «tort» d'arriver précisément le 
31 décembre. Il ne reste plus qu'à espérer que le glissement de sens dû à la traduction sera rattrapé par le bon sens des autorités qui appliquent le texte. De façon plus générale, la restitution précise du message juridique suppose une bonne compréhension de l'interaction entre le texte et le système dans lequel il s'intègre. C'est la sous-compétence thématique et culturelle qui peut livrer les éléments nécessaires, en permettant de saisir les liens avec d'autres textes et de tenir compte des pratiques d'interprétation et d'application des règles (voir Šarčević 1997: 60-61, 74; Sparer 2002: 272).

Quant à la pluralité des destinataires potentiels, que nous avons également identifiée comme caractéristique de la traduction juridique dans les contextes de plurilinguisme officiel, elle a des conséquences qui sont rarement mentionnées. Elle rend nécessaire la maîtrise de plusieurs registres, au-delà de celui qui s'adresse au spécialiste. Elle exige aussi une capacité de rédiger avec clarté (voir Sparer 2002: 275). Cette dernière ne va pas de soi compte tenu de l'environnement dans lequel, souvent, les traducteurs travaillent. Les contraintes discursives imposées par le cadre institutionnel, la situation minoritaire dans un milieu de travail plurilingue et, dans certains cas, l'appartenance à une communauté linguistique "périphérique» peuvent être génératrices d'une certaine insécurité linguistique (voir Francard 1993) et d'un sentiment de résignation à l'égard des interférences (faux amis, calques, etc.) qui «brouillent» la communication. Un renforcement de la sous-compétence communicative et textuelle peut apporter une réponse à ces difficultés, moins connues des traducteurs qui travaillent dans un environnement social plus unilingue et pour un public de spécialistes.

Enfin, l'institutionnalisation de la traduction dans les contextes de plurilinguisme officiel amène les traducteurs à collaborer régulièrement avec d'autres professionnels. En l'occurrence, il ne s'agit pas seulement de commenter le «produit fini», en expliquant à posteriori tel ou tel choix de traduction au donneur d'ouvrage. Il s'agit d'intégrer la traduction dans un processus interdisciplinaire complexe (voir Šarčević 1997: 95-100). Plus qu'ailleurs, les traducteurs juridiques doivent être à même d'expliquer les opérations qu'ils accomplissent et les critères sur lesquels ils se fondent, dans un langage factuel, précis, mais aussi accessible à des interlocuteurs ayant une autre formation. L'élaboration des textes juridiques plurilingues confère aux traducteurs, en tant que groupe professionnel, une part de responsabilité quant au rapport entre institutions et citoyens et à l'évolution de la langue. C'est la sous-compétence interpersonnelle et professionnelle qui joue ici un rôle particulier.

TABLEAU 1

Caractéristiques de la traduction juridique dans les contextes de plurilinguisme officiel: implications pour les compétences du traducteur

\begin{tabular}{|l|l|}
\hline Intertextualité et notions uniformes & $\begin{array}{l}\text { Renforcement de la sous-compétence documentaire et } \\
\text { technologique, qui reste dépendante de la sous-compétence } \\
\text { stratégique/méthodologique }\end{array}$ \\
\hline Texte cible = instrument juridique & $\begin{array}{l}\text { Priorités dans le développement de la sous-compétence } \\
\text { thématique et culturelle }\end{array}$ \\
\hline Destinataires multiples & $\begin{array}{l}\text { Renforcement ciblé de la sous-compétence communicative } \\
\text { et textuelle }\end{array}$ \\
\hline Institutionnalisation du processus & $\begin{array}{l}\text { Renforcement ciblé de la sous-compétence interpersonnelle } \\
\text { et professionnelle }\end{array}$ \\
\hline
\end{tabular}




\section{Mettre l'accent sur certains éléments de compétence : propositions pour la formation des traducteurs}

\subsection{Une situation professionnelle à intégrer dans la formation}

Quelles sont les implications de ce qui précède pour la formation des traducteurs? La problématique du plurilinguisme officiel présente assez de spécificités pour mériter d'être abordée à l'université, dans le cadre d'un enseignement de la traduction juridique articulé autour des situations professionnelles. En d'autres termes, il ne suffit pas de former à la traduction juridique "en général», en considérant que les éléments méthodologiques propres à la pratique des institutions plurilingues seront acquis ensuite «sur le tas» (dans le même ordre d'idées, voir Sosoni 2011). Il est bien entendu que la conception d'un programme en traduction juridique tient compte de nombreux paramètres, parmi lesquels le contexte géographique et le cadre dans lesquels se déroule la formation. Cependant, la mobilité géographique et professionnelle suppose aujourd'hui une faculté d'adaptation à des situations professionnelles diverses. L'acquisition de cette faculté figure parmi les objectifs d'une formation universitaire en traduction.

\subsection{Préparer une utilisation raisonnée des ressources documentaires}

Dans le traitement du plurilinguisme institutionnel, il importe que le volet documentaire et terminologique de la formation aille au-delà d'une présentation des ressources, pour examiner en profondeur leur mise en œuvre dans la perspective de la traduction.

Ainsi, la portée du phénomène de l'intertextualité peut être illustrée par les problèmes que pose la formulation des renvois. Selon, au sens de, aux termes de, conformément à, en vertu de, en application de... Quelle locution choisir? Serait-il préférable d'opter pour une reformulation globale (par exemple, "[l'acte] prévu par l'article...») ? Ces choix supposent que le traducteur saisisse avec précision le rapport logique sous-jacent, car les langues source et cible ne le verbalisent pas nécessairement de la même manière et n'en explicitent pas forcément le même aspect. Signaler le risque de faux sens, voire de contresens, aide à prendre conscience de la nécessité de se documenter en consultant les sources juridiques.

Pour ce qui est de la terminologie, il importe d'apprendre à dissocier l'étape de recherche documentaire du choix de traduction proprement dit, lequel prend en considération des paramètres contextuels et situationnels complexes. Une bonne base méthodologique peut être donnée par la comparaison de problèmes représentant divers cas de figure tels qu'une terminologie officielle incohérente, contradictoire ou inexistante, ou encore un terme apparaissant dans des types de textes différents. Voici trois exemples tirés du contexte suisse.

(1) Terme officiel problématique:

Dans le canton de Berne (canton bilingue où l'on traduit principalement d'allemand en français), le terme Alkoholabgabe a pour équivalent officiel redevance d'alcool. Il désigne une «redevance perçue par le Canton pour les autorisations qui comprennent le droit de servir ou de vendre des boissons alcooliques, qui est versée au Fonds de lutte contre les toxicomanies de la Direction de la santé publique et de la prévoyance sociale, aux fins de lutter contre l'alcoolisme ${ }^{4} »$. En français, la construc- 
tion du terme est problématique à cause de l'imprécision du rapport logique exprimé par la préposition de. La consultation d'un certain nombre de sources francophones extérieures au canton de Berne met sur la piste d'autres équivalents possibles: redevance sur/grevant/frappant l'alcool / la vente d'alcool. Le traducteur qui exerce son activité dans le contexte bernois doit-il dans tous les cas reprendre le terme officiel? Ou peut-il nuancer ses choix terminologiques, par exemple entre la traduction d'une motion parlementaire et celle d'un texte de vulgarisation destiné au secteur de la restauration? Voire entre plusieurs occurrences du terme dans le même texte?

(2) Notion marginale:

À l'échelon fédéral, l'unification de la procédure pénale a été réalisée très récemment, avec l'adoption d'un code en 2007 et son entrée en vigueur en 2011. Le Message relatif à l'unification du droit de la procédure pénale du 21 décembre $2005^{5}$, qui accompagnait le projet de code, fait référence à quelques instruments procéduraux, existant dans d'autres systèmes juridiques, qui ont été écartés. Parmi eux, le recours à la déposition d'un complice à qui la justice accorde, en échange de sa collaboration, un allégement de sa propre peine. Le texte allemand emploie le terme Kronzeuge pour désigner cette figure, connue entre autres dans les systèmes anglosaxons ainsi qu'en Italie (pentito, «repenti», dans le contexte de la lutte contre la criminalité organisée). Une recherche documentaire dans divers dictionnaires et banques de terminologie aboutit à une liste impressionnante d'équivalents français plus ou moins approximatifs, parmi lesquels «témoin transfuge ${ }^{6} »$ dans le contexte canadien. Le choix d'une stratégie dépendra directement, ici, du cadre très particulier dans lequel le terme apparaît: la notion est seulement évoquée à l'occasion d'une réforme, elle est présentée comme une notion étrangère et elle est destinée à le rester; il ne s'agit donc pas de créer un nouveau terme officiel appelé à «fonctionner» en droit suisse.

(3) Variations liées à la répartition des compétences dans le cadre du fédéralisme:

Si la procédure pénale (et aussi civile) a été unifiée récemment, l’organisation judiciaire continue à relever, dans une large mesure, des cantons. Chacun a donc ses propres noms de tribunaux dans ses propres langues officielles. Le canton de Thurgovie (germanophone) est doté par exemple de Bezirksgerichte et d'un Obergericht. Au civil, leurs homologues s'appellent Tribunaux d'arrondissement et Tribunal cantonal dans le canton de Vaud, mais Tribunal de premiere instance et Cour de justice dans le canton de Genève, pour ne prendre que deux exemples de cantons francophones. Entre emprunt, traduction littérale, équivalent fonctionnel, périphrase explicative, quel procédé (ou combinaison de procédés) choisir? La décision ne sera pas la même selon qu'il s'agit de traduire une pièce de procédure pour un juge vaudois ou un arrêt du Tribunal fédéral pour une revue de jurisprudence.

Un cas encore plus complexe est celui de la dénomination des autorités communales: dans ce domaine, les phénomènes de synonymie mais aussi de polysémie créent des risques de confusion à l'intérieur d'une même langue. Certaines communes ont un législatif distinct de l'exécutif, tandis que d'autres sont dotées d'un organe unique, et le même terme (p. ex. Stadtrat) est susceptible de recouvrir des notions différentes selon le lieu.

\subsection{Présenter le cadre juridique}

Sur le plan des connaissances thématiques, il est utile d'intégrer dans la formation des questions de droit linguistique et de fonctionnement institutionnel. Selon quel 
régime le plurilinguisme officiel est-il organisé? Comment est-il intégré dans la production des textes normatifs, par exemple aux différentes étapes du processus législatif? Comment est-il intégré dans l'application de ces mêmes textes, à travers la procédure judiciaire et administrative?

Une nouvelle fois, la démarche comparative peut se révéler très fructueuse car elle rend sensible à l'influence de certains facteurs. Voici un exemple d'exercice, tiré du contexte suisse, qui invite à situer l'opération de traduction en prenant en considération le cadre juridique dans lequel le texte cible sera appelé à «fonctionner». Les étudiants se voient proposer les deux cas suivants:

- Cas 1:

L'Office fédéral de l'agriculture (OFAG) [une subdivision de l'administration fédérale] a attribué à une société installée dans un canton francophone une part de contingent tarifaire partiel pour l'importation de poudre de lait. La société a payé le prix d'adjudication avec un certain retard. Elle reconnaît avoir commis une erreur. L'OFAG décide de prendre une mesure administrative (il s'agit, en l'occurrence, d'un "avertissement»). Le texte source de la décision, qui émane du service juridique, comporte des parties en français (éléments standardisés; extraits de l'échange de correspondance préalable) et des parties en allemand, qui doivent être traduites.

- Cas 2:

Une société d'assurances ayant son siège à Zurich est en conflit avec une assurée, domiciliée dans le canton de Genève, à propos de la prise en charge d'un médicament par l'assurance-maladie obligatoire. L'assurée a fait recours contre une décision de la société en saisissant la Chambre des assurances sociales de la Cour de justice du canton de Genève. La société s'apprête à déposer un mémoire en réponse. Le texte source, qui émane du service juridique, est rédigé en allemand mais cite en français quelques extraits d'échanges antérieurs.

Dans chaque cas, un extrait anonymisé du texte source est fourni aux étudiants. L'exercice consiste d'abord en une analyse comparative de ces deux situations, selon la grille suivante:

- Pourquoi ce texte peut-il être qualifié de «juridique»?

- De quel type de texte juridique s'agit-il?

- Comment l'acte de communication se situe-t-il par rapport aux différents paliers du fédéralisme suisse?

- Pourquoi le texte doit-il être traduit?

- Quel sera le statut du texte cible?

- Pour le traducteur, quels sont les principaux objectifs et problèmes?

À cette première étape succédera un travail approfondi sur la traduction de l'extrait.

L'analyse comparative permet de dégager les éléments suivants:

- Juridicité:

Les deux textes contribuent à la réalisation du droit par l'application de ses normes, le premier directement, le second en tant qu'élément à prendre en considération dans une décision future (voir Cornu 1990/2005: 209).

- Type de texte:

Le premier texte est une décision administrative. Le second, un écrit émanant d'une partie à une procédure judiciaire. Tous deux sont de type argumentatif et s'inscrivent dans un déroulement complexe, comportant plusieurs «épisodes». 


\section{- Place dans le fédéralisme:}

Dans le cas 1, une autorité fédérale communique avec une entreprise privée. Dans le cas 2 , une entreprise privée est partie à une procédure devant une instance cantonale.

- Nécessité de traduire:

Dans le cas 1, le plurilinguisme officiel de la Confédération oblige l'autorité à employer, pour communiquer avec l'entreprise, la langue officielle choisie par cette dernière. Dans le cas 2, l'unilinguisme officiel du canton de Genève fait du français la langue de procédure. Dans les deux cas, il aurait été tout à fait possible de rédiger le texte directement en français, sans passer par la traduction. S'il se trouve que le rédacteur initial est germanophone, c'est en raison du fonctionnement interne de l'administration/de l'entreprise (implantation géographique et recrutement du personnel). En d'autres termes, la nécessité de la traduction naît de l'interaction entre, d'une part, les régimes linguistiques «de droit» dans un contexte fédéral et, d'autre part, la réalité sociolinguistique.

- Statut du texte cible:

Dans les deux cas, c'est le texte cible qui aura valeur juridique. L'existence du texte source est contingente.

- Principaux objectifs et problèmes:

Les deux textes s'inscrivent dans une situation conflictuelle qui présente des risques, d'où une exigence de grande précision. Les deux textes sont fournis aux traducteurs sous la forme d'un enchevêtrement de passages en allemand et de passages en français, sans qu'il soit toujours précisé si ces derniers doivent être révisés. Ces documents de départ ne correspondent pas à la notion classique de «texte source».

Cette analyse étant faite, le travail peut se concentrer sur quelques points: les références plus ou moins explicites à des documents antérieurs liés à chaque affaire, ainsi que les renvois aux sources législatives, jurisprudentielles et doctrinales; la progression thématique et les connecteurs, éléments essentiels pour la clarté de l'argumentation. Il se trouve que chacun des textes pose, par ailleurs, des problèmes terminologiques et phraséologiques assez représentatifs. Le premier texte fait appel à des notions élastiques: dans quelle mesure le retard de paiement est-il justifié par un "empêchement non fautif», l'entreprise a-t-elle fait preuve de la "diligence requise», les lettres de rappel qu'elle a reçues de l'administration contenaient-elles des «informations claires»? Ces expressions doivent-elles être traitées comme des collocations occasionnelles ou comme de véritables termes, correspondant à une notion définie par la loi et/ou délimitée par la jurisprudence? Quant au second texte, il fait appel à la notion d' «essai/étude clinique», dont la dénomination et la définition présentent quelques incertitudes. En permettant d'identifier le texte et de saisir les enjeux de sa traduction, l'analyse comparative préalable aide à trouver des pistes pour résoudre ces divers problèmes.

Sur l'autre versant de la communication juridique, celui de la réception, il est particulièrement intéressant d'illustrer l'interaction entre les choix de traduction (ou de rédaction plurilingue) et l'interprétation des textes, en puisant dans la jurisprudence. Il existe en effet un certain nombre de décisions qui font entrer en jeu des problèmes de divergence - au moins apparente - entre les textes d'une loi dans ses différentes langues (voir Cao 2007: 122-133). Elles permettent de montrer de façon assez frappante que la «marge de manœuvre» interlinguistique est non négligeable, mais connaît des limites. Elles permettent surtout de souligner la difficulté de prévoir 
les problèmes de lecture qui seront soulevés au fil du temps par les cas d'espèce, ce qui invite à être prudent dans la reformulation du message.

Pour ce dernier point, un exemple intéressant peut être donné à l'aide d'un arrêt du Tribunal fédéral suisse datant de 1944 (ATF 70 II 17). Les juges devaient se pencher sur un cas de mariage fictif à la suite duquel l'épouse demandait le divorce. Elle se fondait sur l'article 142, alinéa premier, du Code civil suisse (divorce pour «causes indéterminées»), tel qu'il était alors en vigueur :

(4) a. Ist eine so tiefe Zerrüttung des ehelichen Verhältnisses eingetreten, dass den Ehegatten die Fortsetzung der ehelichen Gemeinschaft nicht zugemutet werden darf, so kann jeder Ehegatte auf Scheidung klagen.

(Schweizerisches Zivilgesetzbuch 1908: 62; nos italiques)

b. Chacun des époux peut demander le divorce lorsque le lien conjugal est si profondément atteint que la vie commune est devenue insupportable.

(Code civil suisse 1908: 62; nos italiques) ${ }^{9}$

c. Ognuno dei coniugi può domandare il divorzio quando le relazioni coniugali siano così profondamente turbate e scosse che non si possa ragionevolmente esigere da essi la continuazione dell'unione coniugale.

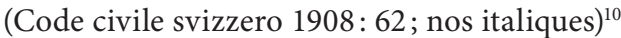

Les juridictions de première instance et d'appel, situées dans un canton francophone (Genève), avaient rejeté la demande. Selon elles, il n'était pas possible de prononcer le divorce en vertu de cet article, puisqu'il n'y avait jamais eu de vie commune. Le Tribunal fédéral a écarté l'argument en relevant que les versions allemande et italienne se référaient à la notion moins restrictive de «communauté conjugale»: on peut imaginer des cas dans lesquels, même en l'absence de "vie commune», il y aurait «communauté conjugale». Le Tribunal fédéral a néanmoins rejeté le recours formé par l'épouse, en invoquant un autre argument: le principe de la bonne foi. Du point de vue jurisprudentiel, son raisonnement voit plus loin que le cas d'espèce: il vise à laisser ouverte la possibilité de se fonder sur l'article en question pour prononcer le divorce dans d'autres cas (mariage non fictif) où il y aurait communauté conjugale sans vie commune. Du point de vue de la traduction, on peut retenir ceci: le changement de perspective observable dans la version française correspond à un procédé couramment employé et rarement problématique; un problème peut toutefois surgir le jour où une affaire atypique vient précisément mettre en lumière la zone de divergence entre les formulations choisies dans les différentes langues.

\subsection{Prévenir les interférences et le jargon}

Sur le plan communicatif et textuel, le renforcement des compétences peut passer par des exercices "antijargon». Ces activités d'appoint sont construites par l'enseignant, à partir d'exemples tirés de la pratique mais selon une logique "pédagogique», pour compléter des activités principales plus représentatives de la réalité professionnelle. Elles se prêtent à un travail ciblé sur les interférences qui constituent des pièges classiques dans tel ou tel couple de langues. Elles permettent également de développer les capacités de reformulation.

Nous donnerons ici quelques exemples, mis au point pour la formation en traduction juridique allemand-français dans le contexte suisse: 


\section{- Traitement des phrases complexes:}

Pour répondre au besoin de précision et de précaution du langage juridique, la langue allemande peut puiser dans de riches ressources morphosyntaxiques (larges possibilités de nominalisation, de subordination, etc.). La densité et la complexité des phrases comptent même parmi les principales difficultés de l'allemand juridique pour les traducteurs de langue française. Le travail sur une sélection de phrases «à tiroirs» - chacune étant présentée dans son contexte - donne l'occasion de mettre en évidence les procédés qui peuvent être mobilisés pour aboutir en français à un texte lisible.

- Traitement du discours rapporté dans les textes de décision:

La langue allemande dispose d'un mode (le subjonctif I) qui permet de déployer un discours indirect sur plusieurs phrases sans avoir à en réexpliciter la nature à travers des verbes introducteurs (du type «il dit...»). Cette ressource est abondamment utilisée dans les décisions (judiciaires ou administratives) pour rapporter les affirmations des parties. Le français étant dépourvu de mode équivalent, le traducteur a le choix, schématiquement, entre l'explicitation (ajouter des verbes introducteurs) et l'implicitation (se contenter de l'indicatif, lorsque le contexte permet de comprendre que le discours indirect se prolonge). Le traducteur peut être tenté de recourir à une autre ressource, à savoir l'emploi du conditionnel. Or la connotation de ce mode donne l'impression que l'autorité met d'emblée en doute les affirmations des parties (ce que, justement, le mode allemand permet d'éviter): dans une décision pénale, traduire "X [der Beschuldigte] habe nicht gewusst, dass $\mathrm{Y}$ und $\mathrm{Z}$ Banküberfälle begingen » par "X [le prévenu] aurait ignoré que $\mathrm{Y}$ et $\mathrm{Z}$ attaquaient des banques", c'est sous-entendre "selon ses dires, auxquels le tribunal accorde peu de crédit». Si le procédé se répète, il risque de donner l'image d'une justice partiale.

- Expression de la généralité (traitement de wer):

En allemand, la proposition introduite par wer est une façon courante d'exprimer la généralité dans les textes normatifs. En français, les calques qui, celui qui et quiconque sont problématiques: le premier évoque le registre du proverbe; les deux autres peuvent avoir une connotation accusatrice. Il est donc nécessaire de reformuler en faisant appel à d'autres moyens d'expression de la généralité (nous donnerons un exemple au paragraphe suivant).

Pour chacun de ces trois cas, une série d'exemples sert de base à un travail approfondi, portant sur les solutions possibles et les critères de choix.

Il s'agit de dépasser la focalisation sur les problèmes terminologiques, travers fréquent dans l'apprentissage de la traduction juridique, pour apprendre à mobiliser les ressources phraséologiques, syntaxiques et textuelles susceptibles de favoriser la clarté du message. À cet égard, il importe de souligner qu'un problème initialement défini comme "terminologique» peut être résolu à un niveau supérieur, l'absence de terme dans la langue cible étant palliée par l'emploi d'une périphrase ou devenant sans importance du fait d'un changement de perspective. Les traductions officielles de bonne qualité ne manquent pas pour faire observer - à posteriori - ce type d'équivalences globales. Il importe par ailleurs de souligner que, si les problèmes terminologiques sont considérables en traduction juridique, c'est souvent une négligence à l'égard des autres dimensions du discours qui aboutit à un manque de clarté. Les exercices qui demandent plusieurs reformulations d'un même passage (voir Šarčević 1997: 162-164) amènent à prendre conscience du fait que, même dans un type de discours très codifié, on peut souvent dire autrement. Ils peuvent porter par exemple sur les éléments appartenant à la structure de base de l'énoncé normatif, à savoir 
l'expression de l'hypothèse, de la généralité et de l'obligation (voir Šarčević 1997: 164-178). Dans l'énoncé de conditions générales ${ }^{11}$, outre l'équivalence officielle (a.), on pourrait imaginer au moins deux autres formulations (b. et c.):

(5) Wer dem Auftraggeber ein Angebot einreicht (Auftragnehmerin), akzeptiert damit vorliegende AGB, soweit in der Offertanfrage keine Abweichungen vorgesehen sind. Änderungen und Ergänzungen vorliegender AGB bedürfen der schriftlichen Vereinbarung.

a. À moins que la demande d'offres n'en dispose autrement, tout mandataire qui présente une offre à un mandant accepte les présentes CG. Ces dernières ne peuvent être modifiées ou complétées que moyennant un accord écrit.

b. Si un mandataire présente une offre à un mandant, il accepte les présentes CG, sauf disposition contraire figurant dans la demande d'offres. Tout changement ou complément apporté aux présentes CG nécessite un accord écrit.

(notre traduction)

c. La présentation d'une offre à un mandant emporte, pour le mandataire, acceptation des présentes CG, sauf exception prévue par la demande d'offres. Un accord écrit est nécessaire pour modifier ou compléter les présentes CG.

(notre traduction)

Les traducteurs céderont moins facilement au transcodage s'ils ont pris l'habitude d'explorer ces possibilités.

Possibilités qui ont certes des limites. Dans un texte de loi destiné à être interprété et appliqué au sein d'un système juridique plurilingue, modifier le découpage en phrases est hasardeux, car les destinataires voudront peut-être se référer par la suite à une phrase précise. À cela viennent s'ajouter les consignes internes qui orientent certains choix de traduction (une politique de féminisation des textes, par exemple). Il appartient aussi à une formation en traduction juridique de signaler l'existence de ces usages et leur influence sur le plan discursif.

\subsection{Apprendre à argumenter et à interagir}

Quant à la capacité d'argumenter et d'interagir avec d'autres professionnels, son développement peut bénéficier d'une didactique faisant appel au travail en groupe. Des ateliers lors desquels un projet de traduction est confié à chaque petit groupe permettent d'apporter un élément d'explicitation et de discussion à toutes les étapes de la démarche: analyse du texte source et de la situation de communication, conduisant à cerner des problèmes et à définir des stratégies (voir Nord 1997: 59-66); recherche documentaire; résolution de problèmes et réexpression du message; relecture; présentation du travail à d'autres groupes et réponse à leurs questions. La révision est un autre type d'activité qui développe les capacités argumentatives, qu'il s'agisse d'une révision "croisée» (entre étudiants) ou de celle d'une traduction réalisée par un tiers.

Le développement de ces capacités peut bénéficier, par ailleurs, d'un enseignement théorique permettant d'acquérir un métalangage. Cet enseignement théorique peut également contribuer à la formation d'une identité professionnelle en intégrant une composante historique: il donne alors l'occasion de montrer que les traducteurs, en tant que groupe actif dans un certain contexte géographique et politique, exercent parfois une influence sur le langage du droit. 


\section{Singularité de chaque contexte plurilingue}

$\mathrm{Au}$-delà des traits communs qui impliquent certaines exigences pour la traduction juridique, chaque contexte de plurilinguisme officiel a ses spécificités (voir Šarčević 2010 et le panorama offert par Borja Albi et Prieto Ramos 2013 : 155-297). Est-il de type régional, national, supranational, international? Comment se présentent les grandes lignes de l'organisation juridique? (A-t-on affaire à une structure de type unitaire ou fédéraliste? comment sont conçus les rapports entre les différentes sources du droit? quelles sont les pratiques interprétatives de la justice et de l'administration?) Qu'en est-il du régime linguistique, autrement dit, du cadre juridique et pratique dans lequel la traduction vient s'insérer: des aménagements institutionnels sont-ils prévus pour intégrer pleinement le plurilinguisme dans les processus d'élaboration et de réception des textes? Enfin, certains aspects socioculturels influencentils les attentes à l'égard de la traduction juridique? Chaque pays ou ensemble plurilingue a une culture politique qui lui est propre. Les relations entre les groupes linguistiques peuvent y revêtir un caractère plus ou moins tendu. Chaque communauté linguistique entretient un rapport culturel à sa langue - et, dans bien des cas, à sa langue au-delà des frontières. Tous ces facteurs, qui débordent largement du domaine juridique, se répercutent sur les habitudes professionnelles à travers des normes traductives transmises de façon plus ou moins explicite. Les nouveaux traducteurs doivent assimiler ces normes (voir Toury 1995/2012: 73-74). Toutefois, une formation de type universitaire doit aussi les rendre capables de poser sur elles un regard critique afin de participer à leur évolution.

Le cadre de la traduction juridique et les pratiques professionnelles sont façonnés par des facteurs qui ne relèvent pas exclusivement du droit. Nous illustrerons cette idée à partir d'une thématique, celle de la lisibilité. Elle constitue une préoccupation commune des divers contextes plurilingues, mais n'est pas abordée partout de la même manière.

$\mathrm{Au}$ Canada, où la traduction juridique a pu être définie comme « un instrument qui contribue à maintenir symboliquement en équilibre une société partagée entre deux langues officielles» (Sparer 2002: 271), les enjeux politiques ont conduit à mettre l'accent sur l'autonomie stylistique du texte de loi dans chaque langue, tandis que la pratique suisse insistait plutôt sur la clarté et l'accessibilité du langage officiel (voir Šarčević 2010 : 42). Il faut souligner qu'en Suisse, le système de la démocratie semidirecte, avec les droits populaires d'initiative et de référendum, a une incidence très concrète sur les textes législatifs: ces derniers sont susceptibles d'être soumis au vote des citoyens, et ce cas de figure n'est pas exceptionnel. La réflexion sur le langage du droit a intégré ce paramètre politique (voir Lötscher et Nussbaumer 2007: 102-104; Schweizer et Borghi 2011 : 345-347) en considérant qu'un langage accessible au profane était souvent souhaitable ou devait, du moins, constituer un horizon (voir Schweizer et Borghi 2011: 371-380).

Dans l'Union européenne, le multilinguisme contribue à rendre la production des textes particulièrement complexe. En conséquence, des efforts sont déployés à grande échelle pour améliorer la qualité des textes officiels. Dans le prolongement de la campagne "Fight the Fog» (Wagner, Bech et al. 2002: 74-75), des conseils et des outils sont diffusés à l'intention de ceux qui rédigent à tous les niveaux ${ }^{12}$.

Aux Nations Unies, les textes juridiques sont élaborés dans un contexte diplomatique. Le degré de précision de leur langage est le résultat d'une négociation. Ainsi, 
le caractère flou de certaines formulations a été voulu par les rédacteurs, et une traduction qui lèverait l'ambiguïté trahirait leur intention (voir Šarčević 1997: 204205; Cao 2007: 135-138, 153). Les pratiques institutionnelles sont donc marquées par la prudence. Pour résumer les contraintes avec lesquelles il faut composer, Cao et Zhao (2008: 48) citent une directive interne datant de 1980:

Writing for the United Nations calls for the same qualities of brevity, clarity, simplicity of language and logical organization of material as are desirable in all writing of a factual character. On the other hand, the need for circumspection in delicate political matters, the tendency to use the jargon of particular specialities and, in many cases, the need to draft in a language other than one's mother tongue militate against such a style. (Editorial directive ST/CS/SER.A/13/Rev.4 of 11 March 1980)

Il reste que la préparation des traducteurs aux particularités du plurilinguisme officiel est une question d'actualité - et un sujet de débat - dans différents contextes institutionnels (voir Sosoni 2011; Cao et Zhao 2008).

\section{Conclusion}

La théorie de la traduction juridique met traditionnellement en relief la complexité des problèmes de droit comparé et de contrastes discursifs qui se posent lors du passage d'un système à un autre (par exemple, lorsqu'un acte notarié provenant de tel État unilingue doit être traduit pour un tribunal d'un autre État unilingue, saisi d'une affaire qui présente un élément d'extranéité). Cette optique a le mérite de dégager les traits qui sont essentiels pour saisir la spécificité de la traduction juridique dans le champ des traductions spécialisées. Elle court toutefois le risque de faire apparaître les contextes de plurilinguisme officiel comme une situation simplifiée, demandant principalement aux traducteurs la maîtrise d'outils spécifiques.

Nous avons montré que, même dans ces contextes, les traducteurs ne pouvaient pas faire l'économie des compétences permettant de résoudre les problèmes classiques de la traduction juridique. Nous avons aussi établi que d'autres compétences devaient être renforcées: la capacité de dissocier ses langues pour reformuler le message dans un discours à la fois clair et précis est sollicitée de façon particulière dans les contextes de plurilinguisme officiel. Le traitement des difficultés propres aux textes juridiques doit pouvoir s'appuyer ici sur une base généraliste solide, complétée par des exercices spécialisés. "A competent legal translator is first of all a competent translator», écrit Deborah Cao (2007: 39). Dans une perspective plus large, les traducteurs doivent développer une sensibilité accrue à des facteurs multiples - linguistiques, juridiques, institutionnels, mais aussi socioculturels - au-delà des compétences strictement «techniques». Enfin, le travail dans un contexte de plurilinguisme officiel suppose souvent une capacité de trouver sa place et de faire valoir son point de vue dans un processus institutionnel.

L'enjeu est une pratique de la traduction qui contribue au bon fonctionnement du système juridique dans des sociétés démocratiques assumant la pluralité des langues (voir Ost 2009: 379-417). Il faut enfin souligner que ceux qui traduisent les textes juridiques dans les contextes de plurilinguisme officiel ne sont pas seulement les gardiens d'un langage statique et les passeurs entre ses divers idiomes, mais interviennent aussi en tant qu'acteurs dans la transformation de ce langage. 


\section{NOTES}

1. Voir Cosmai 2003/2007: 11-18 à propos de l'occultation du rôle de la traduction, et 114-116 à propos de son interaction avec l'activité des juristes-linguistes dans l'Union européenne. Pour un aperçu de la place actuelle des traducteurs dans le processus complexe d'élaboration des lois plurilingues en Suisse, voir le site des Services linguistiques centraux de la Chancellerie fédérale (consulté le 13 mars 2014, www.bk.admin.ch/org/bk/00332/00337/index.html ?lang=fr) et les articles rédigés par des traducteurs à partir de leur expérience, p. ex. Bertagnollo et Laurent 2005, Egger et Grandi 2008. Voir aussi Schweizer et Borghi 2011: 30-42. Ce livre résulte d'un travail interdisciplinaire effectué dans le cadre du Programme national de recherche 56, «Diversité des langues et compétences linguistiques en Suisse». Les chercheurs ont suivi la genèse de la Loi fédérale sur la formation professionnelle (2002), menant en particulier des entretiens avec des traducteurs. (Rapport final du 22 décembre 2008 consulté le 13 mars 2014. www.nfp56.ch/f_projekt. $\mathrm{cfm}$ ? Slanguage $=\mathrm{f} \& \mathrm{kati}=3 \&$ Projects. . Command $=$ details\&get $=15)$

2. Norme européenne EN 15038:2006 (F), Services de traduction - Exigences requises pour la prestation du service, adoptée par le Comité européen de normalisation, Bruxelles, le 13 avril 2006.

3. Groupe D'experts EMT [European Master's in Translation] (2009): Compétences pour les traducteurs professionnels, experts en communication multilingue et multimédia, Bruxelles. Consulté le 31 janvier 2013, http://ec.europa.eu/dgs/translation/programmes/emt/key_documents/emt_competences_translators_fr.pdf.

4. Voir ce terme dans Lingua-PC Online [banque de terminologie du canton de Berne]. Consulté le 29 juin 2013. www.linguapc.apps.be.ch

5. Publié dans la Feuille fédérale le 7 février 2006. Consulté le 29 juin 2013. www.admin.ch/opc/de/ federal-gazette/2006/1085.pdf pour l'allemand (voir p. 1112-1113), www.admin.ch/opc/fr/federalgazette/2006/1057.pdf pour le français (1086-1087) et www.admin.ch/opc/it/federal-gazette/ 2006/989.pdf pour l'italien (1017-1018).

6. Voir ce terme dans TERMIUM Plus. Consulté le 29 juin 2013. www.termiumplus.gc.ca.

7. Consulté le 29 juin 2013 sur le site de l'Université de Berne, http://servat.unibe.ch/dfr/pdf/c2070001. pdf

8. Schweizerisches Zivilgesetzbuch vom 10. Dezember 1907: Ausgabe in den drei Nationalsprachen (1908). Bern: Francke.

9. Code civil suisse du 10 décembre 1907: édition dans les trois langues nationales (1908). Berne: Francke.

10. Codice civile svizzero del 10 dicembre 1907: edizione nelle tre lingue nazionali (1908). Berna: Francke.

11. Conditions générales de la Confédération pour les services informatiques du 20 octobre 2010, 1.2. Consulté le 29 juin 2013. www.bbl.admin.ch/bkb/02617/02618/02626/index.html?lang=de (allemand) et www.bbl.admin.ch/bkb/02617/02618/02626/index.html?lang=fr (français).

12. Voir par exemple la brochure Rédiger clairement, Commission européenne, mars 2010. Consulté le 29 juin 2013. http://ec.europa.eu/translation/writing/clear_writing/how_to_write_clearly_fr.pdf

\section{RÉFÉRENCES}

Bertagnollo, Fabienne et Laurent, Caroline (2005): Unkraut vergeht nicht. La corédaction dans l'administration fédérale suisse. In: Jean-Claude GÉmAR et Nicholas Kasirer (dir.), Jurilinguistique: entre langues et droits. Montréal/Bruxelles: Thémis/Bruylant, 119-126.

Borja Albi, Anabel et Prieto Ramos, Fernando (dir.) (2013): Legal Translation in Context. Professional Issues and Prospects. Oxford/Bern: Lang.

CAO, Deborah (2007): Translating Law. Clevedon/Buffalo: Multilingual Matters.

CAo, Deborah et Zhao, Xingmin (2008): Translation at the United Nations as Specialized Translation. JoSTrans. 9:39-54. Consulté le 29 juin 2013. www.jostrans.org/issue09/art_cao. pdf

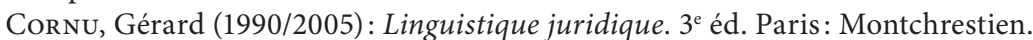

Cosmai, Domenico (2003/2007): Tradurre per l'Unione europea: prassi, problemi e prospettive del multilinguismo comunitario dopo l'ampliamento a est. $2^{\mathrm{e}}$ éd. Milano: Hoepli. 
EgGer, Jean-Luc et Grandi, Filippo (2008): Il nuovo Codice di procedura penale: un cantiere anche linguistico. LeGes. 19(1):31-72. Consulté le 13 mars 2014. www.bk.admin.ch/themen/ lang/00938/02124/04296/index.html?lang=fr

Francard, Michel (dir.) (1993): L'insécurité linguistique dans les communautés francophones périphériques (colloque de Louvain-la-Neuve, 10-12 novembre 1993). Vol. 1. Leuven/Louvainla-Neuve: Peeters/Institut de linguistique.

Kelly, Dorothy A. (2002): Un modelo de competencia traductora: bases para el diseño curricular. Puentes. 1:9-20.

KJaer, Anne Lise (2007): Legal Translation in the European Union: A Research Field in Need of a New Approach. In: Krzysztof KRedens et Stanisław Goźdź-RoszkowsKi (dir.), Language and the Law: International Outlooks. Frankfurt a.M.: Lang, 69-95.

Koskinen, Kaisa (2011): Institutional translation. In: Handbook of Translation Studies online. Consulté le 29 juin 2013. http://benjamins.com/online/hts

Lavoie, Judith (2003): Faut-il être juriste ou traducteur pour traduire le droit? Meta. 48(3):393401. Voir aussi le courrier des lecteurs dans Meta. 49(2):457-458.

Lötscher, Andreas et Nussbaumer, Markus (dir.) (2007): Denken wie ein Philosoph und Schreiben wie ein Bauer. Sprache, mit der ein Staat zu machen ist. Zürich: Schulthess.

Nord, Christiane (1997): Translating as a Purposeful Activity. Functionalist Approaches Explained. Manchester: St. Jerome.

Ost, François (2009): Traduire. Défense et illustration du multilinguisme. Paris: Fayard.

PACTE (2003): Building a translation competence model. In: Fabio Alves (dir.), Triangulating Translation. Perspectives in Process-oriented Research. Amsterdam/Philadelphia: Benjamins, 43-66.

PACTE (2005): Investigating Translation Competence: Conceptual and Methodological Issues. Meta. 50(2):609-619.

Prieto Ramos, Fernando (2011): Developing legal translation competence. An integrative process-oriented approach. Comparative Legilinguistics. 5:7-21.

RAUs, Rachele (dir.) (2010): Multilinguismo e terminologia nell'Unione europea: problematiche e prospettive. Milano: Hoepli.

SANDRINI, Peter (2010): Rechtsübersetzen in der EU: Translatio legis pluribus. In: Lew N. Zyватоw (dir.), Translationswissenschaft - Stand und Perspektiven. Frankfurt a.M. : Lang, 143-157.

ŠArČEvić, Susan (1997): New Approach to Legal Translation. The Hague: Kluwer.

ŠARČEvić, Susan (2010): Legal translation in multilingual settings. In: Icíar Alonso Araguás et al. Translating Justice. Granada: Comares, 19-45.

SCARPA, Federica (2001/2008): La traduzione specializzata. Un approccio didattico professionale. $2^{\mathrm{e}}$ éd. Milano: Hoepli.

Schäffner, Christina et ADAB, Berverly (dir.) (2000): Developing Translation Competence. Amsterdam/Philadelphia: Benjamins.

Schweizer, Rainer J. et Borghi, Marco (dir.) (2011): Législation plurilingue en Suisse. Étude juridique et linguistique de textes légaux plurilingues de la Confédération et des cantons. Zurich: Dike.

Sosoni, Vilelmini (2011): Training translators to work for the EU institutions: Luxury or necessity? JoSTrans. 16: 77-108. Consulté le 29 juin 2013. www.jostrans.org/issue16/art_ sosoni.pdf

Sparer, Michel (2002): Peut-on faire de la traduction juridique? Comment doit-on l'enseigner? Meta. 47(2):266-278.

Toury, Gideon (1995/2012): Descriptive Translation Studies - and Beyond. Éd. rev. Amsterdam/ Philadelphia: Benjamins.

Tranchant, Isabelle (2011): Les méthodes de traduction et la terminologie juridique: l'expérience du département de langue française de la Commission européenne. In: Xavier NoRTH (dir.), Traduction du droit et droit de la traduction. (Université de Poitiers, 15-16 octobre 2009.) Paris: Dalloz, 199-206. 
WAGNER, Emma, Bech, Svend, et al. (2002): Translating for the European Union Institutions. Manchester: St. Jerome.

Wiesmann, Eva (2009): Rechtstexte - eine übersetzerische Herausforderung und ihre didaktischen Konsequenzen. In: Piergiulio TAINo et al. Eindeutig uneindeutig: Fachsprachen ihre Übersetzung, ihre Didaktik. Frankfurt a.M.: Lang, 11-26. 